\title{
RELIABILITY PREDICTION OF THICK FILM HYBRID INTEGRATED CIRCUITS
}

\author{
RUMEN PRANCHOV, VENETA ATHANASSOVA, RUSLAN \\ GEORGIEV \\ Technical University Sofia, Department of Electronics Technology, Sofia 1756, Bulgaria \\ STEFAN POMAKOV \\ Plant for Capacitors, Kyustendil 2500, Bulgaria \\ (Received July 12, 1991; in final form October 23, 1991)
}

\begin{abstract}
When using the well known handbooks for reliability predicting (MIL, CNET) some problems occur in quality assessment of components which are not produced according to the requirements of the US military or the French standards. The results obtained from tests of Test Pattern Circuits may be used successfully for reliability estimating during the design period of new thick film Hybrid Integrated Circuits. Six types of Test Pattern Circuits were designed and tested and a model for estimating thick film Hybrid Integrated Circuits failure rate is proposed. Failure rate values predicted by using the method with Test Pattern Circuits, and the results, obtained from the tests of two kinds of Hybrid Integrated Circuits concerned for a $90 \%$ confidence level were compared.
\end{abstract}

Key Words: Reliability, Prediction methods, Thick film, ICs

\section{INTRODUCTION}

The increasing complexity of thick film Hybrid Integrated Circuits (HIC), the widening range of their functional abilities and the decreasing of their design period make direct reliability testing insufficiently effective or often unacceptable. Difficulties, not only of an economic, but of a purely technological nature occur. It is very difficult or even impossible to analyse the failure reasons of a complex circuit. The failure data, obtained from long-term tests reveal extremely high stability of the thick film structures in particular and the HIC in general.

The complex assessment of technological features of thick film HIC may be made through evaluation of their reliability characteristics. The influence of the technology parameters and used materials are not considered in the well known handbooks for reliability predicting (MIL, CNET) $)^{1,2}$. Some problems occur in quality assessment of components which are not produced according to the requirements of the US military or the French standards. These problems could be solved by using Test Pattern Circuits (TPC), which are subjected to reliability testing. The results obtained are projected on the actual HIC, making reliability assessment more correct. This approach is based on reliability characteristics estimation of structure elements with critical behaviour in reliability aspects and produced via 
the same technological process and from the same materials as the actual HIC. For better confidence the TPC are comprised of a large number of identical structure elements.

\section{DESIGN OF THE TEST PATTERN CIRCUITS}

In the development of TPC for structure reliability prediction the following problems occur:

- Composing the list of elements to be tested,

- Design of the topology and the construction of the TPC,

- Defining the test conditions,

- Choosing methods for measuring the controlled parameters.

The list of elements, composing different TPCs is chosen after complete analysis of the failure mechanisms in thick film $\mathrm{HICs}^{3,4,5}$. Failure mechanisms can be classified in two groups-intrinsic and extrinsic mechanisms.

Sources of intrinsic failure mechanisms are the elements comprising the thick film HIC: resistors (degradation), cross-overs of thick film conductors separated by dielectric pad (migration of metal ions), active components (degradation, breakdown) and packed capacitors (migration of metal ions). Sources of extrinsic failure mechanisms are: packaging (accelerates film resistors degradation, disturbs thermal conditions) and interconnections (cracks, due to thermal mismatch between substrate and chip-components, interdiffusion, electromigration, corrosion and contaminations)

After analysing the nature of the failure mechanisms and the results from many long-term tests, six TPC were designed (Fig. 1):

- TPC 1-3 identical chip transistors,

- TPC 2-9 identical thick film resistors,

- TPC 3-25 identical cross-overs of two groups of 5 thick film conductors each,

- TPC 4-18 identical interconnections,

- TPC 5-9 identical chip-diodes,

- TPC 6-5 identical chip-capacitors.

In order to obtain correct information from the results of the TPC tests, it is important to design optimum test conditions. Because of the thick film HIC high stability, sufficient information for a reasonable period of time may be obtained only through accelerated tests.

The main factor subject to acceleration is the temperature. Test temperature maximum value is limited by the material of lowest thermal stability and by the requirement for preserving the nature of the failure causing physical processes, $\left(E_{a}\left(T_{o}\right)=\right.$ const. $)$. As far as the electrical stress is concerned, usually the maximum level allowed is applied. 

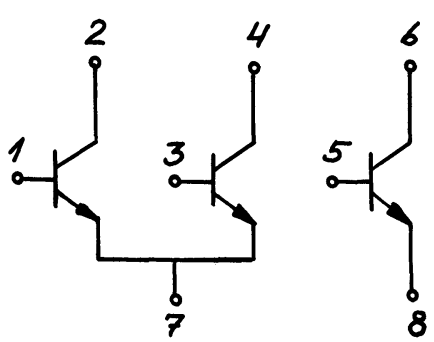

TPC 1

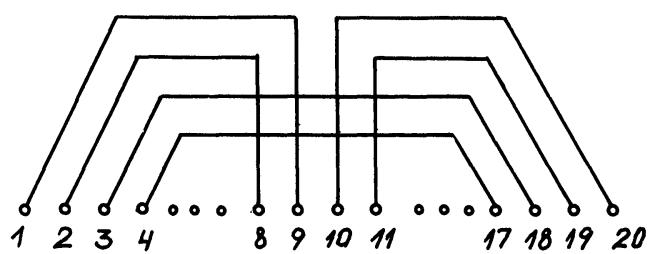

$T P C_{3}$



TPC 5

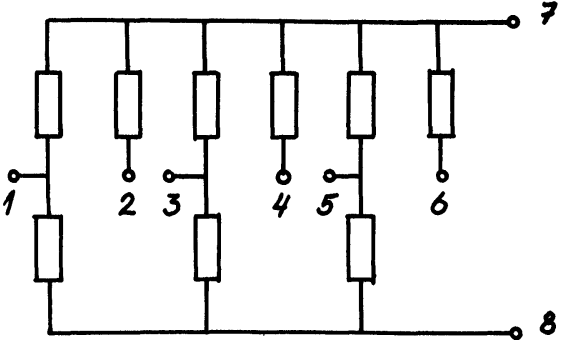

TPC 2



$\operatorname{TPC} 4$



TPC 6

FIGURE 1 Base Types of Test Pattern Circuits (TPC). 


\section{MODEL FOR THICK FILM HIC RELIABILITY PREDICTION}

The model for estimating thick film HIC failure rate is

$\lambda_{\mathrm{HIC}}=\left[N_{a} \lambda_{a}+N_{c} \lambda_{c}+\left(N_{c o} \lambda_{c o}+N_{i} \lambda_{i}+N_{R} \lambda_{R}\right) \pi_{F}\right] \pi_{D}$

where: $N_{a}$ and $\lambda_{a}$ are the number and the failure rate of the active components;

$N_{c}$ and $\lambda_{c}$ are the number and the failure rate of the chip-capacitors;

$N_{c o}$ and $\lambda_{c o}$ are the number and the failure rate of the cross-overs of thick film conductors, separated by a dielectric pad;

$N_{i}$ and $\lambda_{i}$ are the number and the failure rate of the interconnections;

$N_{R}$ and $\lambda_{R}$ are the number and the failure rate of the thick film resistors;

$\pi_{F}$ is the circuit function factor;

$\pi_{D}$ is the density factor $\left(\pi_{F}\right.$ and $\pi_{D}$ are estimated according to MIL-HDBK-217D recommendations).

\section{EXPERIMENTAL RESULTS}

The designed TPC were subjected to accelerated tests. The $\chi^{2}$-test was applied to determine whether the results obtained would be accepted as coming from exponential distributions. The calculated values of $\chi^{2}$ from the data were less than the corresponding cumulative probability values of $p=90 \%$ on the $\chi^{2}$-distribution and hence the hypotheses of exponential distributions of the data were accepted at the level of significance postulated. For example the calculated value of $\chi^{2}$ from the data of the tests of TPC 3 of 9.968 was less than the corresponding value of $\chi^{2}=$ 10.645 for $p=0.9$ and 6 degrees of freedom so the hypothesis of an exponential distribution was accepted at $10 \%$ level of significance.

The results obtained from the tests were used for estimation of the failure rates (Table I).

Long-term tests of two types of thick film HIC-A and B were performed. These circuits are of normal complexity: type $\mathrm{A}$ includes 3 diodes, 1 transistor, 1 chip capacitor, 13 film resistors, 11 pins; and type B: 5 diodes, 9 film resistors and 10 pins. Table II shows failure rate values predicted by using the method with TPCs and the results obtained from the tests of the circuits concerned for a $90 \%$ confidence level.

TABLE I

Failure rates of test pattern circuits

\begin{tabular}{lcc}
\hline Components & $\lambda, 1 / \mathrm{h}$ \\
\hline Resistor & $30.10^{-10}$ \\
Interconnection & $2.10^{-10}$ \\
Cross-over & $2.10^{-9}$ \\
Chip-capacitor & $1,5.10^{-9}$ \\
Chip-diode & $1,5.10^{-9}$ \\
Chip-transistor & $2.10^{-8}$ \\
\hline
\end{tabular}


TABLE II

Estimated values of failure rates, $1 / \mathrm{h}$

\begin{tabular}{lll}
\hline Circuit type & Predicted reliability & Test reliability \\
\hline A & $7,1.10^{-8}$ & $3,3.10^{-8}$ \\
B & $4,4.10^{-8}$ & $2,5.10^{-8}$ \\
\hline
\end{tabular}

\section{CONCLUSIONS}

The model for thick film HIC reliability prediction, based on the results from TPC tests provides the opportunity to evaluate reliability characteristics during the design period. This model allows one to reveal the actual technology and material peculiarities. The experimental results from the tests of two kinds of hybrid integrated circuits and the predicted values show a good coincidence.

\section{REFERENCES}

1. Reliability Prediction of Electronic Equipment, MIL-HDBK-217D.

2. Recueil de donnees de fiabilite du CNET (1979).

3. F.N. Sinnadurai, P.E. Spencer and K.J. Wilson, "Some observations on the accelerated ageing of thick film resistors." Electrocomp. Sci. and Tech., 6, pp. 241-246 (1980).

4. M. Ciez, "Failure mechanisms in thick-film layers." Proc. 4th European Hybrid Microelectr. Conf., pp. 1-11 (1983).

5. M. Coleman, "Ageing Mechanisms and stability in thick film resistors." Proc. 4th European Hybrid Microelectr. Conf., pp. 20-30 (1983). 

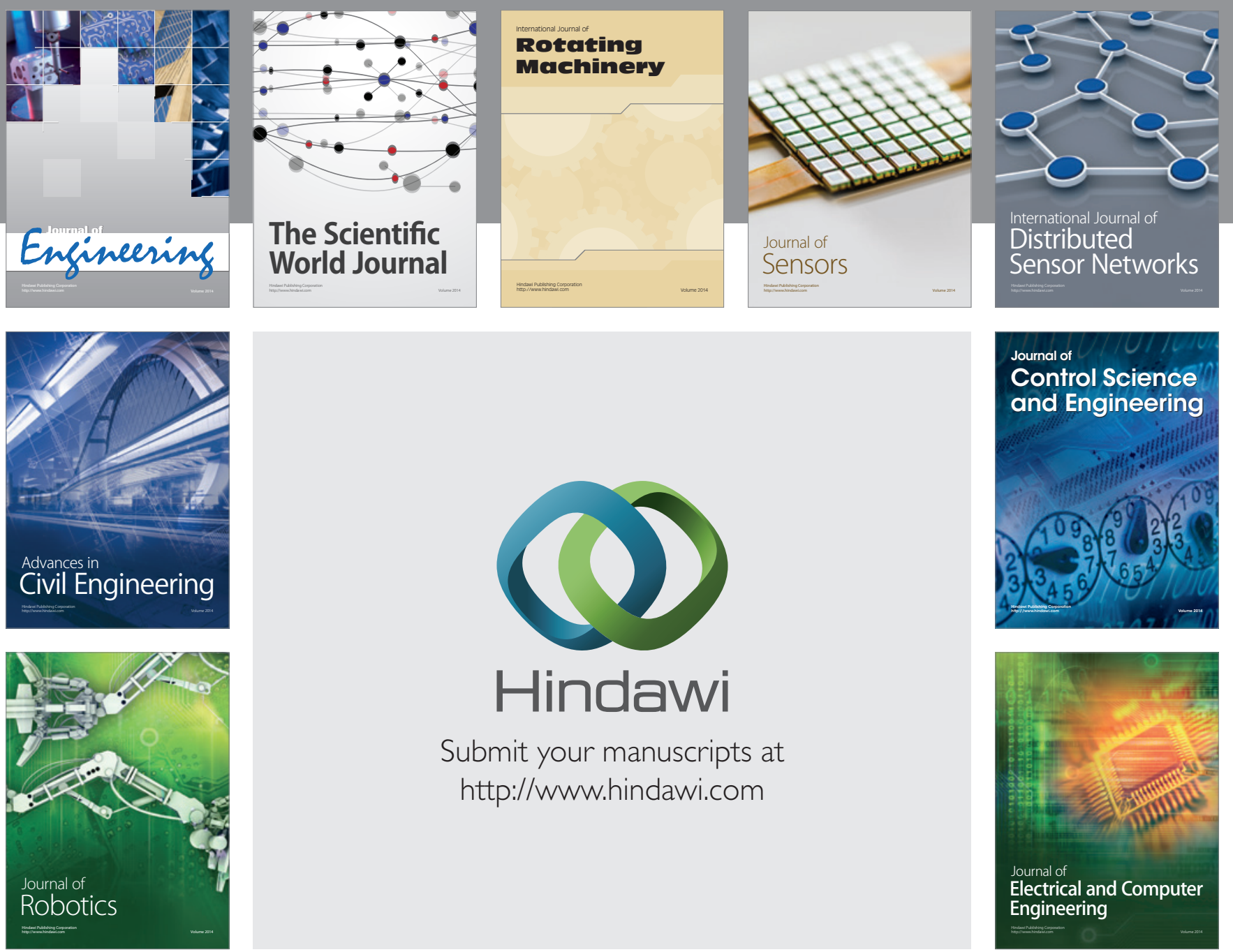

Submit your manuscripts at

http://www.hindawi.com
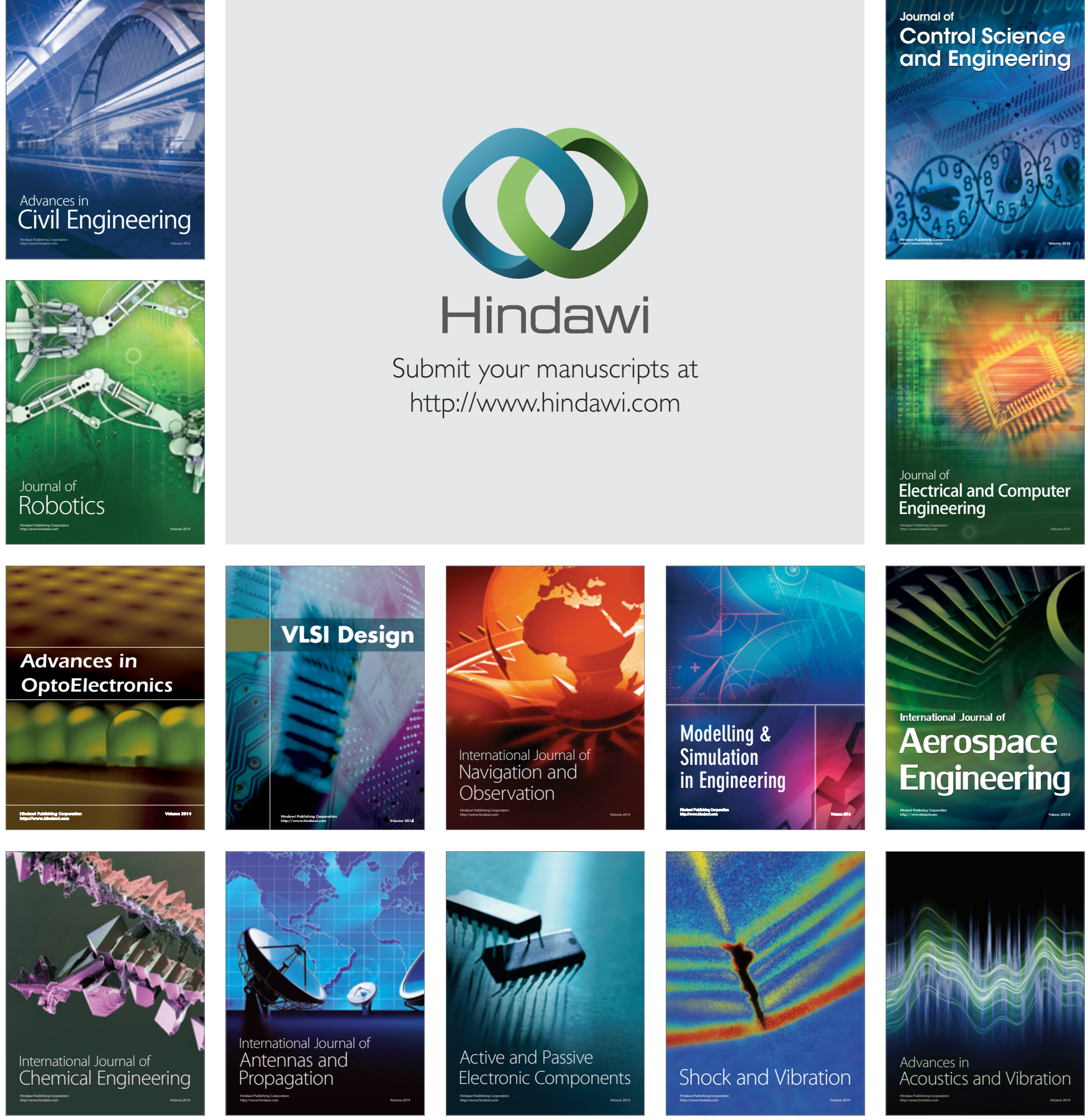